\title{
XXXIV. A hydrodynamical proof of the equations of motion of a perforated solid, with applications to the motion of a fine rigid framework in circulating liquid
}

\section{G. H. Bryan}

To cite this article: G. H. Bryan (1893) XXXIV. A hydrodynamical proof of the equations of motion of a perforated solid, with applications to the motion of a fine rigid

framework in circulating liquid, Philosophical Magazine Series 5, 35:215, 338-354, DOI: 10.1080/14786449308620417

To link to this article: http://dx.doi.org/10.1080/14786449308620417

$$
\text { 曲 Published online: } 08 \text { May } 2009 .
$$

Submit your article to this journal ¿

\section{山 Article views: 2}

Q View related articles ¿ 
XXXIV. A Hydrodynamical Proof of the Equations of Motion of a Perforated Solid, with Applications to the Motion of a Fine Rigid Framework in Circulating Liquid. By G. H. BRYAN*.

\section{Introduction.}

1. TN the whole range of hydrodynamics, there is probably no investigation which presents so many difficulties as that which deals with the equations of motion of a perforated solid in liquid. The object of the present paper is to show how these equations may be deduced directly from the pressure-equation of hydrodynamics, without having recourse to the laborious method of ignoration of coordinates. The possibility of doing this is mentioned by Prof. Lamb in his 'Treatise on the Motion of Fluids' (pp. 119, 120), but he dismisses the method with the brief remark that in most cases it would prove exceedingly tedious. I think, however, that it will be admitted that the following investigation is more straightforward and simple than that given by Basset in his 'Hydrodynamics,' vol. i. pp. 167-178.

The usual method presents little difficulty when the motion of the liquid is acyclic, because the whole motion could in such cases be set up from rest by suitable impulses applied to the solids alone; and a consideration of Routh's modified Lagrangian function shows that in this case the equations of motion can be obtained by expressing the total kinetic energy as a quadratic function of the velocity-components of the solid alone, and applying the generalized equations of motion referred to moving axes.

If, however, the solid is perforated, and the liquid is circulating through the perforations, this method presents several difficulties. If the solid were reduced to rest by the application of suitable impulses, the liquid would still continue to circulate through the perforations, the "circulation" in any circuit remaining unaltered. From this and other circumstances we are led to infer that these circulations are not generalized velocity-components, but rather that the quantities $\kappa \rho$ are generalized momenta. Now the kinetic energy of the system is naturally calculated as a function of the velocity-components of the solid and of these constant circulations (or the corresponding momenta); a form unsuited for obtaining the equations of motion. We ought either to bave the kinetic energy expressed in terms of generalized velocitycomponents alone, or to know the "modified Lagrangian

* Communicated by the Physical Society : read February 24, 1893. 
function" obtained by "ignoring" the velocity-components corresponding to the constant momenta or circulations. Either of these expressions involves constants which cannot be determined from the ordinary expression for the energy alone, and to determine them in the usual way it is necessary to resort to arguments based on a consideration of the "impulse" by which the motion might be set up from rest.

In the following investigation the equations of motion are deduced from purely hydrodynamical considerations, and from them the modified function is found. In $\$ \$ 12-16$ the equations of motion are interpreted for the case in which the solid is a light rigid framework and the inertia is entirely due to the circulation of the liquid, and the results are applied to interpret the effective forces of the cyclic motion for a perforated solid in general.

\section{General Hydrodynamical Equations.}

2. Let a perforated solid bounded by the surface $S$ be moving through an infinite mass of liquid (density $\rho$ ) with translational and rotational velocity-components $u, v, w, p, q, r$, referred to axes fixed in the solid, and let $\kappa_{1}, \kappa_{2}, \kappa_{3} \ldots \kappa_{m}$ be the circulations in circuits drawn through the various apertures. Then we know that $\phi$ the velocity-potential of the fluid motion may be expressed as a linear function of the velocities and circulations in the form

$$
\phi=u \phi_{u}+v \phi_{v}+w \phi_{w}+p \phi_{p}+q \phi_{q}+r \phi_{r}+\Sigma \kappa \phi_{\kappa}, \quad .
$$

where evidently $\phi_{u}=\partial \phi / \partial u$ \&c., and the coefficients $\phi_{u} \ldots$ depend only on the form of the solid.

If $d v$ denotes the element of the normal to $S$ measured from the solid into the liquid, $(l, m, n)$ its direction-cosines, then, in the usual way, we have

$$
\frac{\partial \phi}{\partial v}=l(u-r y+q z)+m(v-p z+r x)+n(w-q x+p y) .
$$

The six coefficients $\phi_{u} \ldots \phi_{r}$ are single-valued functions of the coordinates, while the coefficients $\phi_{x}$ which determine the part of the velocity-potential due to the circulations are cyclic funetions making $\partial \phi_{\kappa} / \partial \nu=0$ at the surface of the solid; these coefficients are supposed known for each form of solid, although their determination in any given case is generally beyond the range of mathematical analysis.

3. Let $\sigma_{1}, \sigma_{2}, \ldots \sigma_{m}$ be barriers drawn across the perforations ; then, in the usual way, the kinetic energy of the liquid 
is found to be $\mathfrak{T}$ : where

$$
\mathfrak{Z}=-\frac{1}{2} \rho \iint \phi \frac{\partial \phi}{\partial \nu} d \mathbf{S}+\frac{1}{2} \rho \mathbf{\Sigma} \kappa \iint \frac{\partial \phi}{\partial \nu} d \sigma=\mathfrak{I}_{1}+\mathbf{K} .
$$

Here $\mathfrak{I}_{1}$ is a quadratic function of the velocity-components of the solid, and is the kinetic energy when the motion is acyclic, and $K$ is a quadratic function of the circulations.

If the axes were fixed in space, the pressure equation (supposing no forces to act on the liquid) would be

$$
\frac{p_{1}}{\rho}+\frac{\partial \phi}{\partial t}+\frac{1}{2} q_{1}^{2}=\text { const. }
$$

(where $p_{1}=$ pressure, $q_{1}=$ resultant velocity of liquid). Owing to the motion of the axes, however, $\partial \phi / \partial t$ must be replaced by the rate of change of $\phi$ at a fixed point, that is by

$$
\frac{d \phi}{d t}-(u-y r+z q) \frac{\partial \phi}{\partial x}-(v-z p+x r) \frac{\partial \phi}{\partial y}-(w-x q+y p) \frac{\partial \phi}{\partial z},
$$

whence the pressure equation becomes

$$
\begin{array}{r}
\frac{p_{1}}{\rho}+\frac{d \phi}{d t}-(u-y r+z q) \frac{\partial \phi}{\partial x}-(v-z p+x r) \frac{\partial \phi}{\partial y}-(w-x q+y p) \frac{\partial \phi}{\partial z} \\
+\frac{1}{2} q_{1}^{2}=\text { const. . . . . (4) }
\end{array}
$$

The Mutual Reactions between the Solid and Liquid.

4. Let $X_{1}, Y_{1}, Z_{1}, L_{1}, M_{1}, N_{1}$ be the component forces and couples which the solid exerts on the liquid; then we have evidently

$$
\mathrm{X}_{1}=\iint l p_{1} d \mathrm{~S}, \quad \mathrm{~L}_{1}=\iint(n y-m z) p_{1} d \mathrm{~S} . .
$$

To reduce these expressions to the required form, we shall have to resort to repeated applications of Green's formula. Since the velocity-potential $\phi$ is a multiple-valued function, it follows that in transforming volume integrals involving $\phi$ we shall obtain surface integrals over the barriers $\sigma_{1}, \sigma_{2}, \ldots \sigma_{m}$ as well as over $\mathrm{S}$ the surface of the solid. On the other hand, the pressure $p_{1}$ and the velocity-components $\partial \phi / \partial x, \partial \phi / \partial y$, $\partial \phi / \partial z$ are single-valued and do not contribute barrier terms to the surface integrals. Moreover, since the circulations $\kappa$ are independent of the time,

$$
\frac{\partial \phi}{\partial t}=u \phi_{u}+\dot{v} \phi_{v}+\dot{w} \phi_{v}+\dot{p} \phi_{p}+\dot{q} \phi_{q}+\dot{r} \phi_{r} ;
$$

and $\partial \phi / \partial t$ is therefore a single-valued function of the velocitycomponents of the solid satisfying Laplace's equation. 
We also notice that

$$
l=\frac{\partial \phi_{u}}{\partial \nu}, \quad n y-m z=\frac{\partial \phi_{p}}{\partial \nu}, \ldots . . .
$$

as may be at once seen by differentiating (2) with respect to $u$ and $p$ respectively. have

5. Substituting for $p_{1}$ in (5) in terms of the velocities, we

$$
\begin{aligned}
\frac{\mathrm{X}}{\rho}= & -\iint l \frac{d \phi}{d t} d \mathrm{~S} \\
& +\iint\left\{(u-y r+z q) \frac{\partial \phi}{\partial x}+(\text { two similar) }\} l d \mathrm{~S}\right. \\
& -\frac{1}{2} \iint\left\{\left(\frac{\partial \phi}{\partial x}\right)^{2}+\left(\frac{\partial \phi}{\partial y}\right)^{2}+\left(\frac{\partial \phi}{\partial z}\right)^{2}\right\}^{l d \mathrm{~S} . ~ . ~ . ~ . ~ . ~}
\end{aligned}
$$

The first line of this expression is, from (6), equal to

$$
\begin{aligned}
& -\iint \frac{d \phi}{d t} \frac{\partial \phi_{u}}{\partial v} d \mathrm{~S} \\
= & \iiint\left\{\frac{\partial \dot{\phi}}{\partial x} \frac{\partial \phi_{u}}{\partial x}+\frac{\partial \dot{\phi}}{\partial y} \frac{\partial \phi_{u}}{\partial y}+\frac{\partial \dot{\phi}}{\partial z} \frac{\partial \phi_{u}}{\partial z}\right\} d x d y d z
\end{aligned}
$$

by Green's transformation. Remembering that $\phi_{u}$ is independent of the time, this integral, taken throughout the liquid, becomes

$$
\begin{aligned}
& =\frac{d}{d t} \frac{\partial}{\partial u} \iiint \int\left\{\left(\frac{\partial \phi}{\partial x}\right)^{2}+\left(\frac{\partial \phi}{\partial y}\right)^{2}+\left(\frac{\partial \phi}{\partial z}\right)^{2}\right\} d x d y d z \\
& =\frac{1}{\rho} \frac{d}{d t} \frac{\partial \mathfrak{T}}{\partial u}=\frac{1}{\rho} \frac{\partial}{\partial t} \frac{\partial \mathfrak{I}_{1}}{\partial u}
\end{aligned}
$$

By Green's transformation the second line is equal to

$$
\begin{aligned}
& -\iiint \frac{\partial}{\partial x}\left\{\left(u-y r^{2}+z q\right) \frac{\partial \phi}{\partial x}+\text { (two similar) }\right\} d x d y d z \\
= & -\iiint\left(r \frac{\partial \phi}{\partial y}-q \frac{\partial \phi}{\partial z}\right) d x d y d z \\
& -\iiint\left\{(u-y r+z q) \frac{\partial}{\partial x}+\text { (two similar) }\right\} \frac{\partial \phi}{\partial x} d x d y d z
\end{aligned}
$$

which by a second application of Green's transformation becomes 
$=\iint(m r-n q) \phi d S-\Sigma \kappa \iint(m r-n q) d \sigma$

$+\iint\{l(u-y r+z q)+m(v-z p+x r)+n(w-x q+y p)\} \frac{\partial \phi}{\partial x} d \mathbf{S}$

$=\iint(m r-n q) \phi d \mathrm{~S}-\Sigma \kappa \iint(m r-n q) d \sigma+\iint \frac{\partial \phi}{\partial \nu} \frac{\partial \phi}{\partial x} d \mathrm{~S}$

by (2).

Lastly, the third line of (7) is, by Green's transformation,

$=\iiint\left\{\frac{\partial \phi}{\partial x} \frac{\partial^{2} \phi}{\partial x^{2}}+\frac{\partial \phi}{\partial y} \frac{\partial^{2} \phi}{\partial x \partial y}+\frac{\partial \phi}{\partial z} \frac{\partial^{2} \phi}{\partial x \partial z}\right\} d x d y d z$

$=-\iint \frac{\partial \phi}{\partial v} \frac{\partial \phi}{\partial x} d \mathrm{~S}$.

Hence, adding the several terms together, we have

$$
\begin{aligned}
\mathrm{X}_{1} & =\frac{d}{d t} \frac{\partial \mathfrak{S}}{\partial u} \\
& +r\left\{\rho \iint m \phi d \mathrm{~S}-\Sigma \kappa \rho \iint m d \sigma\right\}-q\left\{\rho \int j n \phi d \mathrm{~S}-\Sigma_{\kappa} \rho \iint n d \sigma\right\} .
\end{aligned}
$$

Now by $(6)$,

$\rho \iint m \phi d S=\rho \iint \frac{\partial \phi_{v}}{\partial v} \phi d \mathrm{~S}$

$=\Sigma \kappa \rho \iint \frac{\partial \phi_{v}}{\partial v} d \sigma-\rho \iiint\left\{\frac{\partial \phi}{\partial x} \frac{\partial \phi_{v}}{\partial x}+\frac{\partial \phi}{\partial y} \frac{\partial \phi_{v}}{\partial y}+\frac{\partial \phi}{\partial z} \frac{\partial \phi_{v}}{\partial z}\right\} d x d y d z$

$=\Sigma \kappa \rho \iint \frac{\partial \phi_{v}}{\partial \nu} d \sigma-\frac{\partial}{\partial v} \frac{1}{2} \rho \iiint\left\{\left(\frac{\partial \phi}{\partial x}\right)^{2}+\left(\frac{\partial \phi}{\partial y}\right)^{2}+\left(\frac{\partial \phi}{\partial z}\right)^{2}\right\} d x d y d z$

$=\Sigma \kappa \rho \iint \frac{\partial \phi_{v}}{\partial v} d \sigma-\frac{\partial \mathfrak{I}}{d v}$.

Therefore

$$
\begin{aligned}
\mathrm{X}_{1}=\frac{d}{d t} \frac{\partial \mathfrak{T}}{\partial u} & -r\left\{\frac{\partial \mathfrak{I}}{\partial v}+\Sigma \kappa \rho \iint\left(m-\frac{\partial \phi_{v}}{\partial v}\right) d \sigma\right\} \\
+q & \left\{\frac{\partial \mathfrak{I}}{\partial w}+\Sigma \kappa \rho \iint\left(n-\frac{\partial \phi_{v}}{\partial v}\right) d \sigma\right\} . .
\end{aligned}
$$

6. In like manner we have

$$
\begin{aligned}
\frac{\mathrm{L}_{1}}{\rho}= & -\iint(n y-m z) \frac{d \phi}{d t} d \mathrm{~S} \\
& +\iint\left\{(u-y r+z q) \frac{\partial \phi}{\partial x}+(\text { two similar })\right\}(n y-m z) d \mathrm{~S}
\end{aligned}
$$


$-\frac{1}{2} \iint\left\{\left(\frac{\partial \phi}{\partial x}\right)^{2}+\left(\frac{\partial \phi}{\partial y}\right)^{2}+\left(\frac{\partial \phi}{\partial z}\right)^{2}\right\}(n y-m z) d \mathrm{~S}$

$=-\iint \frac{d \phi}{d t} \frac{\partial \phi_{p}}{\partial v} d \mathrm{~S}$

$-\iiint\left\{y\left(q \frac{\partial \phi}{\partial x}-p \frac{\partial \phi}{\partial y}\right)-z\left(p \frac{\partial \phi}{\partial z}-r \frac{\partial \phi}{\partial x}\right)\right\} d x d y d z$

$-\iiint\left[y\left\{(u-y r+z q) \frac{\partial}{\partial x}+\right.\right.$ (two similar) $\} \frac{\partial \phi}{\partial z}$

$-z\left\{(u-y r+z q) \frac{\partial}{\partial x}+\right.$ (two similar) $\left.\} \frac{\partial \phi}{\partial y}\right] d x d y d z$

$+\iiint\left(\frac{\partial \phi}{\partial x} \frac{\partial}{\partial x}+\frac{\partial \phi}{\partial y} \frac{\partial}{\partial y}+\frac{\partial \phi}{\partial z} \frac{\partial}{\partial z}\right)\left(y \frac{\partial \phi}{\partial z}-z \frac{\partial \phi}{\partial y}\right) d x d y d z$

$=\frac{1}{\rho} \frac{d}{d t} \frac{\partial \mathfrak{I}}{\partial p}$

$+\iint\left\{l(u-y r+z q)+(\right.$ two sim. $\left.)-\frac{\partial \phi}{\partial v}\right\}\left(y \frac{\partial \phi}{\partial z}-z \frac{\partial \phi}{\partial y}\right) l \mathrm{~S}$

$-\iiint\left[y\left(q \frac{\partial \phi}{\partial x}-p \frac{\partial \phi}{\partial y}\right)-z\left(p \frac{\partial \phi}{\partial z}-r \frac{\partial \phi}{\partial x}\right)\right.$

$\left.-(v-z p+w v) \frac{\partial \phi}{\partial z}+(w-x q+y p) \frac{\partial \phi}{\partial y}\right] d x d y d z$.

Remembering that in this expression one factor of the surface integral is zero at every point of $S$, we have, by again applying Green's transformation to the volume integral,

$$
\begin{aligned}
\mathrm{L}_{1}= & \frac{d}{d t} \frac{\partial \mathfrak{I}}{\partial p} \\
& +w\left\{\rho \iint m \phi d \mathrm{~S}-\Sigma_{\kappa} \kappa \iint n d \sigma\right\}-v\left\{\rho \iint n \phi d \mathrm{~S}-\Sigma \kappa \rho \iint n d \sigma\right\}
\end{aligned}
$$$$
+r\left\{\rho \int j(l z-n x) \phi d \mathrm{~S}-\Sigma \kappa \rho \iint(l z-n x) d \sigma\right\}
$$$$
-q\left\{\rho \iint(m x-l y) \phi d S-\Sigma \kappa \rho \iint(m x-l y) d \sigma\right\} .
$$

Now, just as before,

$\rho \iint(l z-m x) \phi d \mathbb{S}=\rho \iint \frac{\partial \phi_{q}}{\partial \nu} \phi d \mathrm{~S}$

$=\Sigma \kappa \rho \iint \frac{\partial \phi_{q}}{\partial v} d \sigma$

$-\rho \iiint\left(\frac{\partial \phi}{\partial x} \frac{\partial \phi_{q}}{\partial x}+\frac{\partial \phi}{\partial y} \frac{\partial \phi_{q}}{\partial y}+\frac{\partial \phi}{\partial z} \frac{\partial \phi_{q}}{\partial z}\right) d x d y d z$

$=\Sigma \kappa \rho \iint \frac{\partial \phi_{q}}{\partial \nu} d \sigma-\frac{\partial \mathfrak{I}}{\partial q}$. 
Therefore,

$$
\begin{aligned}
\mathrm{L}_{1}= & \frac{d}{d t} \frac{\partial \mathfrak{I}}{\partial p} \\
& -w\left\{\frac{\partial \mathfrak{I}}{\partial v}+\Sigma \kappa \rho \iint\left(m-\frac{\partial \phi_{v}}{\partial v}\right) d \sigma\right\} \\
& +v\left\{\frac{\partial \mathfrak{I}}{\partial w}+\Sigma \kappa \rho \iint\left(n-\frac{\partial \phi_{w}}{\partial v}\right) d \sigma\right\} \\
& -r\left\{\frac{\partial \mathfrak{I}}{\partial q}+\Sigma \kappa \rho \iint\left(l z-n x-\frac{\partial \phi_{q}}{\partial v}\right) d \sigma\right\} \\
& +q\left\{\frac{\partial \mathfrak{I}}{\partial r}+\Sigma \kappa \rho \iint\left(m x-l y-\frac{\partial \phi_{r}}{\partial v}\right) d \sigma\right\} .
\end{aligned}
$$

Application to the Equations of Motion.

7. The equations of motion of the solid may now be written down at once. Let $\mathfrak{S}^{\prime}$ be the kinetic energy of the solid, $\mathrm{T}$ the total kinetic energy $=\mathfrak{L}+\mathfrak{I}^{\prime}$; and suppose that the motion takes place under the action of a system of external impressed forces and couples designated by X, Y, Z, L, M, N. Then the effective forces and couples to which the motion of the solid itself is due are $X-X_{1} \ldots, L-L_{1} \ldots$, respectively, and the six equations of motion of the solid referred to the moving axes are of the form

$$
\begin{gathered}
\frac{d}{d t} \frac{\partial \mathfrak{I}^{\prime}}{\partial u}-r \frac{\partial \mathfrak{I}^{\prime}}{\partial v}+q \frac{\partial \mathfrak{I}^{\prime}}{\partial w}=\mathrm{X}-\mathrm{X}_{1}, \ldots . \\
\frac{d}{d t} \frac{\partial \mathfrak{I}^{\prime}}{\partial p}-w \frac{\partial \mathfrak{I}^{\prime}}{\partial v}+v \frac{\partial \mathfrak{I}^{\prime}}{\partial w}-r \frac{\partial \mathfrak{I}^{\prime}}{\partial q}+q \frac{\partial \mathfrak{I}^{\prime}}{\partial r}=\mathrm{L}-\mathrm{L}_{1} .
\end{gathered}
$$

Hence, on substitution, we see that the required equations of motion are found by writing $T$ for $\mathfrak{T}$ and $X, Y, Z, L, M, N$ for $X_{1}, Y_{1}, Z_{1}, L_{1}, M_{1}, N_{1}$ in equations (9) (11). The resulting equations may be written :

$$
\begin{aligned}
\mathrm{X}=\frac{d}{d t} \frac{\partial \mathrm{T}}{\partial u}-r\left(\frac{\partial \mathrm{T}}{\partial v}+\eta\right) & +q\left(\frac{\partial \mathrm{T}}{\partial w}+\zeta\right), . . . \\
\mathrm{L}=\frac{d}{d t} \frac{\partial \mathrm{T}}{\partial p}-w\left(\frac{\partial \mathrm{T}}{\partial v}+\eta\right)+ & +v\left(\frac{\partial \mathrm{T}}{\partial w}+\zeta\right) \\
& -r\left(\frac{\partial \mathrm{T}}{\partial q}+\mu\right)+q\left(\frac{\partial \mathrm{T}}{\partial r}+v\right),
\end{aligned}
$$

where $\xi, \eta, \zeta, \lambda, \mu, \nu$ are defined by the equations 


$$
\begin{aligned}
& \xi=\Sigma \kappa \rho \iint\left(l-\frac{\partial \phi_{u}}{\partial \nu}\right) d \sigma, \& \mathrm{c} . \quad . . . . \\
& \lambda=\Sigma \kappa \rho \iint\left(n y-m z-\frac{\partial \phi_{p}}{\partial \nu}\right) d \sigma, \& \mathrm{c} . \quad . \quad .
\end{aligned}
$$

As Lamb has pointed out ('Motion of Fluids,' p. 140), the six quantities $(\xi, \eta, \zeta, \lambda, \mu, \nu)$ are "the components of the impulse of the cyclic fluid motion which remains when the solid is (by forces applied to it alone) brought to rest" *. They are linear functions of the circulations and their form depends on the form of the solid. If there is only one aperture they are all proportional to the cireculation $\kappa$.

\section{The Modified Lagrangian Function.}

8. We shall now show that the motion of the solid can be determined in terms of Routh's modified Lagrangian function, and shall find the form of this function for the system. Putting

$$
\mathrm{H}=\mathrm{T}+\xi u+\eta v+\zeta v+\lambda p+\mu q+v r+\mathrm{F}(\kappa \rho), .
$$

where $F(\kappa \rho)$ denotes any function whatever of the quantities $\kappa \rho$, we see that the equations of motion reduce to the standard form

$$
\begin{aligned}
& \mathrm{X}=\frac{d}{d t} \frac{\partial \mathrm{H}}{\partial u}-r \frac{\partial \mathrm{H}}{\partial v}+q \frac{\partial \mathrm{H}}{\partial w}, . . . \\
& \mathrm{L}=\frac{d}{d t} \frac{\partial \mathrm{H}}{\partial p}-w \frac{\partial \mathrm{H}}{\partial v}+v \frac{\partial \mathrm{H}}{\partial v}-r \frac{\partial \mathrm{H}}{\partial q}+q \frac{\partial \mathrm{H}}{\partial r} . .
\end{aligned}
$$

The function $\mathrm{H}$, therefore, plays the same part in determining the equations of motion of the solid as the kinetic energy $\mathrm{T}$ in the case of an imperforated solid (or any solid when the motion of the liquid is acyclic). It remains (i.) to determine what quantities are to be regarded as the generalized velocities if the quantities $\kappa \rho$ are regarded as generalized momenta ; (ii.) to find the form of the function $F(\kappa \rho)$ in order that $\mathrm{H}$ may represent the modified Lagrangian function.

9 . Let $\dot{\chi}_{m}$ be the generalized velocity-component corresponding to the ignored momentum $\boldsymbol{\kappa}_{m} \rho$. Then, as Routh has shown ('Rigid Dynamics,' vol. i. \$420), the modified Lagrangian function $\mathrm{H}$ is of the form

$$
\mathrm{H}=\mathrm{T}-\Sigma_{\kappa_{m}} \rho \dot{\chi}_{m}, \cdot \cdot \cdot \cdot \cdot \cdot
$$

* Our $\xi, \eta, \zeta, \lambda, \mu, \nu$ are the same as the $\xi_{0}, \eta_{0}, \zeta_{0}, \lambda_{0}, \mu_{0}, \nu_{0}$ of Lamb, or the $\mathfrak{X}, \mathfrak{Y}, \mathfrak{Z}, \mathfrak{l}, \mathfrak{M l}$, $\mathfrak{j} \mathfrak{k}$ of Basset's 'Hydrodynamics.' 
and therefore by equating the two expressions for $\mathrm{H}$ we must have

$$
\xi u+\eta v+\xi_{v}+\lambda p+\mu q+v r+\mathrm{F}(\kappa)=-\Sigma \kappa \rho \dot{\chi} . .
$$

Since $\dot{\chi}_{m}$ is the generalized velocity-component corresponding to the momentum $\kappa_{m} \rho$, therefore

$$
\frac{\partial H}{\partial \cdot \kappa_{m} \rho}=-\dot{\chi}_{m} \cdot \quad \cdot \quad \cdot \quad \cdot .
$$

Now $H$ is a homogeneous quadratic function of the six velocities $(u \ldots, p \ldots)$ and the momenta $\kappa \rho$; therefore

$$
2 \mathrm{H}=\Sigma u \frac{\partial \mathrm{H}}{\partial u}+\Sigma \kappa \rho \frac{\partial \mathrm{H}}{\partial \cdot \kappa \rho}=\Sigma u \frac{\partial \mathrm{H}}{\partial u}-\Sigma \kappa \rho \dot{\chi} .
$$

Hence, from (21),

$$
2 \mathrm{~T}=\Sigma u \frac{\partial \mathrm{H}}{\partial u}+\Sigma_{\kappa} \rho \dot{\chi}=\Sigma u \frac{\partial \mathrm{H}}{\partial u}-\Sigma_{\kappa \rho} \frac{\partial \mathrm{H}}{\partial \cdot \kappa \rho} . .
$$

The portions of $\mathrm{T}$ and $\mathrm{H}$ which involve only the momenta $\kappa \rho$, and are independent of the six velocities $(u \ldots, p \ldots)$, must arise from the terms $\Sigma_{\kappa} \rho \dot{\chi}$ in the above expressions (24) (25), and must therefore be equal and of opposite sign in the expressions $\mathrm{T}$ and $\mathrm{H}$ respectively. Hence, since from (3)

$$
\mathrm{T}=\mathfrak{I}^{\prime}+\mathfrak{\Sigma}_{1}+\mathrm{K}
$$

the portion of $\mathrm{H}$ which is independent of the six velocities $(u \ldots, p, \ldots)$ must be $-\mathrm{K}$, so that

$$
\begin{aligned}
\mathrm{H} & =\mathfrak{T}^{\prime}+\mathfrak{T}_{1}+(\xi u+\eta v+\zeta w+\lambda p+\mu q+\nu r)-\mathrm{K} \\
& =\mathrm{T}+\langle\xi u+\eta v+\zeta v+\lambda p+\mu q+\nu r)-2 \mathrm{~K}, . .
\end{aligned}
$$

and therefore

$$
\mathrm{F}(\kappa \rho)=-2 \mathrm{~K} \text {. . . . . . }
$$

The function $\mathrm{F}(\kappa \rho)$ does not enter into the six equations of motion of the solid, but its form requires to be determined if we wish to reduce the equations of motion of the whole system to the canonical or Hamiltonian form.

\section{The Generalized Velocities and Momenta.}

10. Comparing (21) with (27), we see that

$$
\Sigma_{\kappa \rho} \dot{\chi}=2 \mathrm{~K}-\left(\xi u+\eta v+\zeta_{w}+\lambda p+\mu q+\nu r\right) .
$$

Now equation (3) may be written in the form

$$
\begin{aligned}
2\left(\mathfrak{Z}_{1}+\mathrm{K}\right)= & -\rho \iint \phi \frac{\partial}{\partial v}\left(u \phi_{u}+\ldots+p \phi_{p}+\ldots+\Sigma_{\kappa} \phi_{\kappa}\right) d \mathrm{~S} \\
& +\Sigma_{\kappa} \rho \iint \frac{\partial}{\partial v}\left(u \phi_{u}+\ldots+p \phi_{p}+\ldots+\Sigma_{\kappa} \phi_{\kappa}\right) d \sigma .
\end{aligned}
$$


But by $\S 2, \partial \phi_{k} / \partial \nu=0$ all over the surface $S$ of the solid. Hence, equating the terms independent of the six velocities $(u \ldots, p \ldots)$ on the two sides of $(29)$, we have

But by (16) (17),

$$
2 \mathrm{~K}=\Sigma_{\kappa} \rho \iint \frac{\partial}{\partial \nu}\left(\Sigma_{\kappa} \phi_{\kappa}\right) d \sigma . \quad . \quad . \quad .
$$

$$
\begin{aligned}
\xi u+\eta v & +\zeta w+\lambda p+\mu q+v r \\
= & -\Sigma \kappa \rho \iint \frac{\partial}{\partial v}\left(u \phi_{u}+\ldots+p \phi_{p}+\ldots\right) d \sigma \\
& +\Sigma \kappa \rho \iint\{l u+m v+n w+(n y-m z) p+\ldots\} d \sigma .
\end{aligned}
$$

Hence, by (28) and (30),

$$
\begin{aligned}
\Sigma_{\kappa} \dot{\chi} & =\Sigma_{\kappa} \rho \iint \frac{\partial}{\partial \nu}\left(u \phi_{u}+\ldots+p \phi_{p}+\ldots+\Sigma_{\kappa} \phi_{\kappa}\right) d \sigma \\
& -\Sigma_{\kappa} \rho \iint\{l(u-y r+z q)+m(v-z p+x v)+n(w-x q+y p)\} d \sigma
\end{aligned}
$$

and therefore

$$
\dot{\chi}_{m}=\iint\left(\frac{\partial \phi}{\partial v}-l(u-y r+z q)-(\text { two similar })\right) d \sigma_{m} .
$$

Now $\partial \phi / \partial \nu$ is the velocity of the liquid resolved along the norinal to the barrier $\sigma_{m}^{\prime}$, and

$$
l(u-y r+z q)+m(v-z p+x v)+n(w-x q+y p)
$$

is the velocity of the barrier $\sigma_{m}$ resolved normally to itself at the point $x, y, z$, supposing the barrier to be fixed relatively to the solid. Their difference, therefore, represents the normal relative velocity of the liquid with respect to the barrier.

Hence $\dot{x}_{m}$ represents the total rate of flow of the liquid across the barrier $\sigma_{m}$ relative to the solid; in other words, the generalized velocity corresponding to the ignored momentum $\rho \kappa_{m}$ is the volume of liquid per unit time flowing through the aperture relatively to the solid.

This property is proved in a different way by Basset in his 'Hydrodynamics,' vol. i. page $\mathbf{1 7 6 .}$

\section{The Form of the Modified Function.}

11. It may be interesting to examine a little more closely the effect of the circulations on the motion of a solid.

When the motion of the liquid is acyclic, the kinetic energy is a homogeneous quadratic function of $(u, v, w, p, q, r)$. In general it therefore involves 21 constants, but by a suitable 
choice of axes it is always possible to reduce this number by six, and a further reduction may be effected when the body is symmetrical. When the motion is cyclic the kinetic energy must be replaced by the modified function which in addition contains the seven terms

$$
\xi u+\eta v+\zeta w+\lambda p+\mu q+\nu r-\mathrm{K},
$$

of which the last term does not enter into the equations of motion of the solid. The six coefficients $(\xi, \ldots \lambda, \ldots)$ are linear functions of the circulations, and they remain constant so long as only conservative forces act on the liquid, for the circulations themselves then remain constant. Hence the modified function $\mathrm{H}$ may be regarded as a non-homogeneous quadratic function of the six velocities involving 28 constants, of which 27 enter into the six equations of motion of the solid.

\section{Equations of Motion of a light thin framework of rigid wires.}

12. To take the simplest possible case, let us suppose the solid to consist of a network of infinitely thin rigid massless wires through the meshes of which the liquid is circulating. If the motion of the liquid were acyclic, the wires would simply cut through the liquid without setting it in motion: hence the kinetic energy $\mathfrak{I}^{\prime}+\mathfrak{I}_{1}$ of the acyclic motion vanishes, and the modified function becomes

$$
\mathrm{H}=\xi u+\eta v+\zeta w+\lambda p+\mu q+\nu r-\mathrm{K}, .
$$

a result otherwise evident from the fact that $\mathfrak{T}_{1}$ only involves surface integrals taken over the infinitely small surface of the solid, while $\xi, \eta, \zeta, \lambda, \mu, \nu$ being integrals taken over the finite surfaces of barriers are in general finite.

If we choose as our axis of $x$ the Poinsot's central axis of the impulse whose six components are $\xi, \eta, \zeta, \lambda, \mu, \nu$, the modified function will reduce to the form

$$
\mathrm{H}=\Xi u+\Lambda p-\mathrm{K} \text {. }
$$

If there is only one aperture, $\xi, \eta, \zeta, \lambda, \mu, \nu$ are all proportional to the circulation $\kappa$ and the central axis of the impulse is fixed in position relative to the solid : if there are several apertures the position of the axis depends on the ratios of the circulations through the various apertures, but throughout the motion it in every case remains fixed relatively to the solid.

The six equations of motion (19) (20) now reduce to

$$
\left.\begin{array}{ll}
\mathrm{X}=0, & \mathrm{~L}=0, \\
\mathrm{Y}=r \Xi, & \mathrm{M}=w \Xi+r \Lambda, \\
\mathrm{Z}=-q \Xi, & \mathrm{N}=-v \Xi-q \Lambda .
\end{array}\right\} .
$$


Since these equations do not involve $u$ or $p$, we see that no forces will have to act on the solid in order to naintain a screw motion whose axis coincides with the central axis of the impulse.

13. To interpret the equations still further, let us suppose that $u$ and $p$ are both zero, since they do not enter into the equations of motion. Then the motion whose components are $(0, v, w, 0, q, r)$ consists of two screws whose axes are the axes of $y$ and $z$ respectively, and, by the theory of screws, these are equivalent to a single screw whose axis is a certain straight line intersecting the axis of $x$ and perpendicular to it. We may take this straight line as our axis of $z$, for hitherto we have only fixed the position of the axis of $x$. We have then

$$
v=0, \quad q=0 \text {. }
$$

The equations (34) therefore reduce to

$$
\left.\begin{array}{ll}
\mathrm{X}=0, & \mathrm{~L}=0, \\
\mathrm{Y}=r \Xi, & \mathrm{M}=w \Xi+r \Lambda, \\
\mathrm{Z}=0, & \mathrm{~N}=0 .
\end{array}\right\} . .
$$

Hence the solid is acted on by a wrench $(\mathrm{Y}, \mathrm{M})$ whose axis is the axis of $y$. Thus the axis of the impressed wrench is perpendicular to the central axis of the impulse of the flaid motion, and to the axis of the screw motion of the body.

Let II be the pitch of the impulse, $\approx$ the pitch of the screw motion of the solid, $\mathrm{P}$ the pitch of the impressed wrench, then

$$
\Pi=\frac{\Lambda}{\bar{B}}, \sigma=\frac{w}{r}, \quad \mathrm{P}=\frac{\mathrm{M}}{\overline{\mathrm{Y}}},
$$

and therefore by (35),

$$
\mathrm{P}=\boldsymbol{\sigma}+\Pi \text {. . . . . . . }
$$

is the relation connecting the three pitches.

In particular, if $r=0$ the equations of motion give

$$
\mathrm{Z}=0, \quad \mathrm{M}=w \Xi \text {, }
$$

showing that a couple $\mathbf{M}$ about the axis of $y$ will produce translational motion with velocity $\mathrm{M} / \Xi$ along the axis of $z$.

14. More generally, let the motion be a screw motion about an axis whose inclination to the axis of $x$ is $\theta$ and whose shortest distance from that axis is a. Take this shortest distance as the axis of $y$, and let the screw motion consist of a linear velocity $\nabla$ combined with an angular velocity $\Omega$, the pitch $\nabla / \Omega$ being denoted, as before, by $\varpi$.

Phil. Mag. S. 5. Vol. 35. No. 215. April 1893. 2 B 
It will be readily found that the six components of the screw motion are

$$
\left.\begin{array}{rl}
u=\mathrm{V} \cos \theta+\Omega a \sin \theta, & p=\Omega \cos \theta \\
v=0, & q=0, \\
w=\mathrm{V} \sin \theta-\Omega a \cos \theta, & r=\Omega \sin \theta,
\end{array}\right\} ; .
$$

so that the equations (34) now give

$$
\left.\begin{array}{ll}
\mathrm{X}=0, & \mathrm{~L}=0, \\
\mathbf{Y}=\Omega \Xi \sin \theta, & \mathrm{M}=\mathrm{V} \Xi \sin \theta-\Omega a \Xi \cos \theta+\Omega \Lambda \sin \theta, \\
\mathrm{Z}=0, & \mathrm{~N}=0,
\end{array}\right\} .
$$

The impressed wrench therefore has for its axis the shortest distance between the axis of the screw motion of the solid and the axis of the impulse of the cyclic fluid motion. To find the pitch of the wrench, we have, by division,

that is,

$$
\frac{\mathbf{M}}{\mathbf{Y}}=\frac{\bar{V}}{\Omega}-a \cot \theta+\frac{\Lambda}{\Xi},
$$

$$
\mathrm{P}=\varpi-a \cot \theta+\Pi \text {. }
$$

15. In the case of a fine massless circular ring $\Lambda$ vanishes, or the impulse of the cyclic motion is purely translational. For it is clear that the axis of the ring is the axis of this impulse (the above axis of $x$ ), also the fluid motion will evidently be unaffected by rotating the ring about its axis; and therefore the modified function is independent of the angular velocity $p$.

The equations (34) now become

$$
\left.\begin{array}{ll}
\mathrm{X}=0, & \mathrm{~L}=0, \\
\mathrm{Y}=r \Xi, & \mathrm{M}=w \Xi, \\
\mathrm{Z}=-q \equiv, & \mathrm{N}=-v \Xi .
\end{array}\right\} \cdot \cdot \cdot
$$

Hence a constant force $Y$ along the axis of $y$ causes uniform rotation with angular velocity $\mathrm{Y} / \mathrm{E}$ about the axis of $z$, and a constant couple $M$ about the axis of $y$ causes uniform translational velocity $M / \Xi$ along the axis of $z$.

It is to be noticed that the impressed wrench never does work in the resulting screw motion, in accordance with the principle of Conservation of Energy.

16. The above results show the effective forces produced by circulation of the fluid on any perforated solid whatever. In the general case the modified function contains the quadratic terms $\mathfrak{I}^{\prime}+\mathfrak{Z}_{1}$ in addition to the terms of the first degree considered in the above investigation. If we suppose that the solid is moving in any given manner, the six equations of motion $(19,20)$ determine the components of the impressed wrench (X, Y, Z, L, M, N) necessary to maintain the given 
motion. This impressed wrench may be divided into two parts, one being due to the terms $\mathfrak{T}^{\prime}+\mathfrak{T}_{1}$ in the modified function, the other being due to the terms

$$
\xi u+\eta v+\xi w+\lambda p+\mu q+v r \text {. }
$$

The first portion is the same as if the motion were acyclic, and represents, therefore, the wrench which would have to be impressed on the solid in order to maintain the given motion if there were no circulation. The second part represents the additional wrench which must be applied on account of the circulations, and the equations to determine it are of the forms found above.

We notice, in particular, that if the solid has any screw motion whose axis coincides with the axis of the impulse of the cyclic fluid motion, the latter wrench vanishes; so that the forces required to maintain the motion are unaffected by the circulations. In other cases the additional wrench is about an axis perpendicular to the axis of the impulse. This is true whatever be the form of the solid and the number of the circulations; but, as has already been pointed out, the position of the axis of the impulse relative to the solid is not in general independent of the circulations unless the solid has but a single aperture.

It is probable that these results might be made to furnish mechanical illustrations of certain physical phenomena ; but with these we are not concerned in the present paper.

\section{Note on the foregoing Paper.}

Concerning the proper measurement of the impulse of the cyclic motion, a difficulty arises; for, as Mr. Bryan remarks, this motion cannot be set up from rest by impulses applied to the solid alone. Suppose, however, that we close each perforation by a barrier in the usual way, and let the barriers be acted on by the impulsive pressures $\kappa_{1} \rho, \kappa_{2} \rho, \ldots$ respectively. And instead of these impulsive pressures being due to external forces, suppose that they are due to some immaterial mechanism attached to the solid. In general, an impulsive wrench must act on the solid to keep it at rest, and this wrench is the required impulse of the cyclic motion; for the only other impulses acting on the system are due to the mutual reactions of the solid and fluid, exerted partly over the surface of the solid and partly through the barriers and attached mechanism, and such mutual reactions cannot affect the impulse. The wrench thus found is of course the same as would be obtained by supposing the impulses on the barriers to be due to external impulsive forces, and compounding with these the 
impulse then necessary to hold the solid at rest. This is in agreement with Prof. Lamb's investigation, which Mr. Bryan has quoted.

More generally, if the solid is in motion, and the liquid is also circulating, we may suppose the instantaneous motion to have been set up from rest by an immaterial mechanism connecting the barriers with the solid at the same time that the requisite external impulses act on the solid. The resultant of these last is, as before, the impulse of the whole motion, and is identical with that found by supposing the barriers actuated by impulses from without, and compounding with these the impulse then necessary to give to the solid its instantaneous motion.

The same point may be further illustrated by supposing the circulations $\kappa$ to vary continuously during the motion. To effect this variation we may suppose finite uniform pressures, $\mathrm{P}_{1} \ldots \mathrm{P}_{m}$, to be exerted over certain ideal surfaces which occupy the positions of barriers. The rate of variation $\dot{k}$ of any circulation is given by $\mathrm{P}=\dot{\kappa} \rho$, and in order that it may take place without the direct operation of external forces and couples we may conceive the pressure $P$ to be due, as before, to some highly idealized mechanism attached to the solid. As before, the only forces capable of modifying the impulse are the external forces acting on the solid; and the equations of motion are therefore still to be found by equating the impressed force- and couple-components to the corresponding variations of the "impulse." Since we know the expressions for the impulse-components corresponding to a given instantaneous motion of the solid and given circulations, we have only to remember that in these expressions the $\kappa$ 's are functions of the time, and, just as before, the equations of motion are directly deducible from Hayward's formulæ. Equations (19) (20) of Mr. Bryan's paper will thus be applicable to the present case, provided that in the value of $H$ given by (27) the $\kappa$ 's are allowed to vary.

An investigation proceeding from a consideration of the impulse of the whole motion is not so entirely satisfactory, I think, as the direct method given by Mr. Bryan ; but, at the same time, this brief attempt to interpret the impulse of the cyclic motion may not be without interest.-C. V. Burton.

\section{Note added by the Author.}

Dr. Burton's note is of much value as showing more exactly what is meant by the "impulse" of the motion in the 
ordinary investigations given by Prof. Lamb, and, in a less intelligible form, by Basset.

The equations of motion under finite forces may be deduced by equating the change of momentum in a small time-interval $\delta \dot{t}$ to the impulse of the impressed forces, taking into account the fact that in the interval $\delta t$ the origin has a displacement of translation $(u \delta t, v \delta t, w \delta t)$ and the axes have rotational displacements ( $p \delta t, q \delta t, r \delta t)$, so that the final momenta are referred to a different set of axes to the ori ginal momenta.

The mode of forming the equations of motion is given by Prof. Greenhill (Encyclopcedia Britannica, art. "Hydromechanics ") for the case of acyclic motion, but it is hardly so obvious why in thus forming the equations of motion of a perforated solid, it is necessary to include in the "impulse" terms representing the components of the wrench applied to the barriers as well as to the solid. We may, however, suppose the changes which actually occur in the time $\delta t$ to have been produced as follows :-

1st. Let the solid and fluid be reduced to rest by an impulsive wrench applied to the solid, and transmitted to a series of barriers crossing the perforations. The components of this wrench will be found to be

$$
\frac{\partial \mathrm{T}}{\partial u}+\xi, \& c ., \ldots \frac{\partial \mathrm{T}}{\partial p}+\lambda, \& c . . .
$$

2nd. The barriers being rigidly connected with the solid, let the latter receive small displacements whose translational and rotational components are $(u \delta t, v \delta t, w \delta t, p \delta t, q \delta t, r \delta t)$ and let the solid come to rest in its new position. The fluid will evidently also come to rest, and therefore no impulse will be impressed on the system by this change (as may be otherwise seen by supposing the change to take place very slowly).

3rd. Let the solid be set in motion with velocity-components $(u+\partial u / \partial t . \delta t, \ldots p+\partial p / \partial t . \delta t \ldots)$ referred to the new positions of the axes, and let the circulations $\kappa$ be started in the new position of the solid by a suitable impulsive wrench applied to the solid and transmitted from it to the barriers.

Then the impulse of the impressed forces (components $\mathrm{X} \delta t . ., \mathrm{L} \delta t \ldots$...) is the resultant of the wrenches required to stop the whole system in the first process and to start it again in the third.

It is, therefore, that impulse which must be compounded with the total impulse in the initial position in order to obtain the total impulse in the final position.

Whence Hayward's equations of motion follow at once (as 
shown in Greenhill's article above referred to), and they take the form of the above equations (14), (15).

If we were merely to stop the solid in the first process without stopping the liquid, the cyclic motion would cause the liquid to exert a pressure on the solid in the second process, and the impulse of this pressure would not be zero, but would have to be taken into account in forming the equations of motion. It would be wrong, therefore, to deduce the equations of motion from the impulse applied to the solid alone, as is evident in the analogous case of a solid containing one or more gyrostats.

XXXV. The Magnetic Field of a Circular Current. $B y$ Professor G. M. Mrnonin, M.A.*

CLERK MAXWELL gives a method of drawing the $\cup$ lines of magnetic force due to a circular current ('Electricity and Magnetism,' Art. 702) by means of a series of circles and a series of parallel lines. The object of the following paper is to show how these curves can be described by a slightly different method, and to exhibit the geometrical connexion of the series of circles.

Let $\mathrm{AQBQ}^{\prime}$ be the circular current whose sense is indicated by the arrows, the plane of the circle being that of the paper;

Fig. 1.

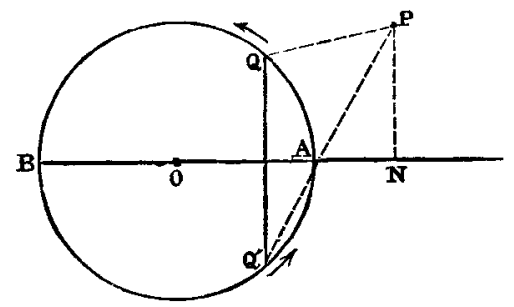

let $\mathrm{P}$ be any point in space, $\mathrm{PN}$ the perpendicular from $\mathrm{P}$ on the plane of the circle, and NAOB the diameter of the circle drawn through $\mathrm{N}$. We shall calculate the vector potential of the current at $P$.

Draw any ordinate, $\mathrm{QQ}^{\prime}$, of the circle perpendicular to $\mathrm{BA}$; and consider two equal elements of length of the circle, each equal to $d s$, at $\mathrm{Q}$ and $\mathrm{Q}^{\prime}$. Resolving each of these along and perpendicular to $\mathrm{QQ}$, we see that the latter components are in opposite senses, and hence their vector potentials at $\mathrm{P}$ cancel

* Communicated by the Physical Society: read March 10, 1893. 